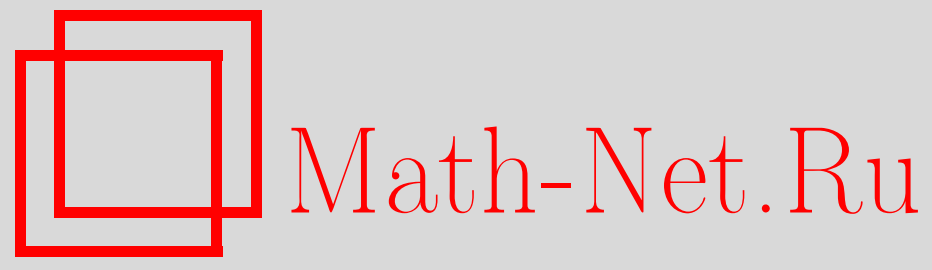

А. С. Пивоварова, Бифуркации в многокомпонентной системе в условиях ограничения информации, Вестн. Сам. гос. техн. ун-та. Сер. Физ.-мат. науки, 2012, выпуск 2(), 152-157

DOI: https://doi.org/10.14498/vsgtu1077

Использование Общероссийского математического портала Math-Net.Ru подразумевает, что вы прочитали и согласны с пользовательским соглашением

http: //www.mathnet.ru/rus/agreement

Параметры загрузки:

IP : 44.207 .124 .84

26 апреля 2023 г., 16:44:58

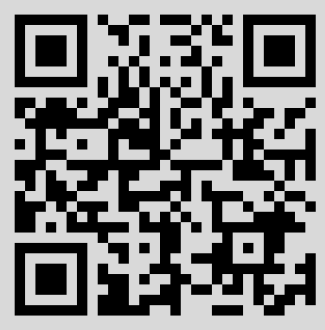


УДК 517.938:519.865.1

\title{
БИФУРКАЦИИ В МНОГОКОМПОНЕНТНОЙ СИСТЕМЕ В УСЛОВИЯХ ОГРАНИЧЕНИЯ ИНФОРМАЦИИ
}

\author{
А. С. Пивоварова \\ Самарский государственный аэрокосмический университет им. ак. С. П. Королёва \\ (национальный исследовательский университет), \\ 443086, Россия, Самара, Московское ш., 34. \\ E-mail: a-pivovarova@mail.ru
}

Предложено многокомпонентное обобщение одномерной модели ВальрасаМариала с функиией предложения, введённой Шананиным. Переход от точечной модели к распределённой осуществляется путём замены одного рынка системой связанных рынков, взаимодействующих посредством функиии спроса. В рамках данной модели исследуются условия установления равновесия для рынка с ограниченной информацией. С помощъю бифуркачионного анализа показывается, что в определённой области параметров системы возникает пространственная неоднородность, качественно меняется сценарий перехода системь $\kappa$ хаосу и смещается положение точки бифуркации.

Ключевые слова: модель равновесия Вальраса-Маршала, распределенная динамическая система, бифуркачионный анализ, устойчивость предельных траекторий, устойчивость состояния равновесия.

Введение. В последние десятилетия для изучения закономерностей поведения рынков активно применяется математический аппарат теории нелинейных динамических систем [1]. Простейшей моделью, описывающей переход рынка от неравновесного состояния к равновесному, является классическая модель Вальраса-Маршала [2], описываемая нелинейным дифференциальным уравнением первого порядка. Различные обобщения данной модели [2-4] позволили исследовать широкий круг явлений, наблюдаемых на реальных рынках. Проблема применения экономических моделей на практике часто связана с ограничениями в распространении информации, т.е. с закрытостью экономики, которая препятствует установлению на рынке единой цены. Это приводит к тому, что переменная цены, входящая в уравнение модели, становится различной для различных агентов. В таких моделях представляет интерес исследование областей параметров, при которых на рынке устанавливается единая цена. В данной работе решается подобная задача в рамках многокомпонентного обобщения классической модели установления рыночного равновесия вальрасовского типа [2] в непрерывном времени, базирующегося на модели формирования предложения, предложенной в [4]. Переход от точечной системы к распределённой основан на введении множества связанных рынков, описывающихся с помощью классической модели ценообразования и взаимодействующих посредством функции спроса. В рамках этой модели численно исследована зависимость асимптотического поведения решений от параметров, таких как относительное число рынков с доступной информацией и эластичность спроса.

Анна Сергеевна Пивоварова, аспирант, каф. физики. 
1. Модель равновесия рынка одного товара. В соответствии с традиционными представлениями теории рыночного равновесия [2] предполагается, что в каждый момент времени товар продаётся по единой цене $p$, а поведение потребителей и производителей описывается функциями спроса $C(p)$ и предложения $Y(p)$. Время предполагается дискретным и $p_{n}$ есть цена на рассматриваемый товар на шаге $n$. Тогда

$$
p_{n}=\frac{p_{n-1} C\left(p_{n-1}\right)}{Y\left(p_{n-1}\right)} .
$$

Непрерывный аналог модели (1) имеет вид

$$
\frac{d p}{d t}=g \frac{C(p)-Y(p)}{Y(p)} p,
$$

где $g>0$ - параметр, имеющий размерность $1 /$ время и характеризующий скорость реакции цены на различие спроса и предложения.

В работе [4] предложен следующий вид функции предложения:

$$
Y\left(p_{n-1}\right)=M(t)\left[1-\left(\frac{s \nu}{p_{n-1}}\right)^{(\gamma+\mu) / \mu}\right],
$$

где $M(t), s>0, \nu>0, \mu>0, \gamma>0$ - параметры модели. В предположении, что спрос на товар не зависит от цены товара, т.е. $C\left(p_{n-1}\right)=C_{0}=$ const, уравнение (1) принимает вид

$$
p_{n}=p_{n-1} \frac{C_{0}}{M(t)}\left[1-\left(\frac{s \nu}{p_{n-1}}\right)^{(\gamma+\mu) / \mu}\right]^{-1} .
$$

Обозначая $x_{n}=s v / p_{n}, \alpha=(\gamma+\mu) / \mu, A(t)=M(t) / C_{0}$, соотношение (2) запишем в виде

$$
x_{n}=A(t)\left(1-x_{n-1}^{\alpha}\right) x_{n-1} .
$$

Для того чтобы данная модель адекватно описывала процесс ценообразования, необходимо, чтобы отображение (3) переводило $x_{n-1} \in[0,1]$ в $x_{n} \in[0,1]$. Такого ограничения можно добиться условием

$$
0 \leqslant A(t) \leqslant \frac{(1+\alpha)^{\frac{1+\alpha}{\alpha}}}{\alpha} .
$$

В работе [4] проведён бифуркационный анализ данной модели. При $\alpha=1$ система (3) представляет собой аналог хорошо изученного в теории динамических систем логистического отображения. Для случая $\alpha=2$ бифуркационная диаграмма по параметру $A$ приведена на рис. 1.

Переход к хаосу в данной модели интерпретируется тем, что увеличение параметра $A=M / C_{0}$ означает увеличение производства по отношению к суммарному спросу, а с ним экономисты связывают кризисы перепроизводства, сопровождающиеся «бурями» в изменении цен.

В описанной модели покупатели планируют объёмы приобретаемой продукции, ориентируясь на цену $p_{n-1}$. Однако предположение о том, что каждому покупателю доступна полная информация о цене $p_{n-1}$, верно только 


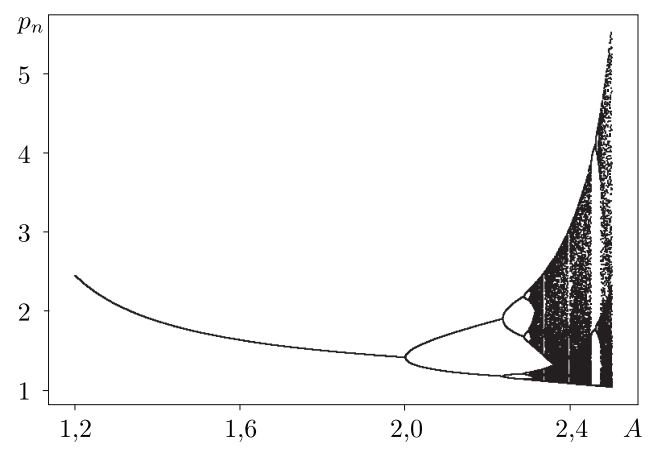

Рис. 1. Бифуркационная диаграмма системы (3) при $\alpha=2$ по параметру $A$

для абсолютно открытых рынков и в большинстве случаев не соответствует реальной ситуации на рынке. Для исследования устойчивости равновесия систем в условиях ограничения информации необходимо рассмотреть многокомпонентную модель, явно учитывающую ограниченный доступ к информации о ценах.

2. Модель рыночного равновесия с учетом отсутствия единой цены на рынке. Для построения многокомпонентной модели рассмотрим множество функций $p^{i}(t)$, где $i=1,2, \ldots M$ - номер рынка. В рамках непрерывной модели для $i$-того рынка функция предложения будет иметь вид

$$
Y\left(p^{i}(t-\tau)\right)=M(t)\left[1-\left(\frac{s \nu}{p(t-\tau)}\right)^{(\gamma+\mu) / \mu}\right],
$$

где $\tau$ - время задержки предложения.

Для оценки текущей рыночной цены покупателями используется равномерное усреднение по выборке из ближайших производителей:

$$
\bar{p}^{i}(t)=\frac{(1+\varepsilon)}{2 k+1} \sum_{j=i-k}^{i+k} p^{j}(t)
$$

где $k$ - радиус корреляции, $\varepsilon-$ случайная величина.

Выберем функцию спроса на $i$-том рынке зависящей от отношения $\chi^{i}(t)=$ $=p^{i}(t) / \bar{p}^{i}(t)$, тем самым учитывая взаимное влияние индивидуальных рынKOB:

$$
C\left(\chi^{i}(t)\right)=C_{0} \operatorname{arctg}\left(e^{\left(p_{\mathrm{cr}}-\chi^{i}(t)\right) E_{\mathrm{c}}}\right),
$$

где $C_{0}$ - нормировочный множитель, определяемый из условия $C(0)=1, E_{\mathrm{c}}$, $p_{\text {cr }}$ - параметры функции спроса.

Группа покупателей $i$-того рынка, ориентируясь на цену $p^{i}(t)$ и среднюю цену на рынке $\bar{p}^{i}(t)$, планирует израсходовать сумму $p^{i}(t) C\left(\chi^{i}(t)\right)$, а производитель планирует выпуск товара в количестве $Y\left(p^{i}(t-\tau)\right)$. В итоге для каждого $i$-того рынка имеем уравнение

$$
\frac{d p^{i}}{d t}=g \frac{C\left(\chi^{i}(t)\right)-Y\left(p^{i}(t-\tau)\right)}{Y\left(p^{i}(t-\tau)\right)} p^{i}(t),
$$

где функции спроса и предложения имеют вид (5) и (4) соответственно. 

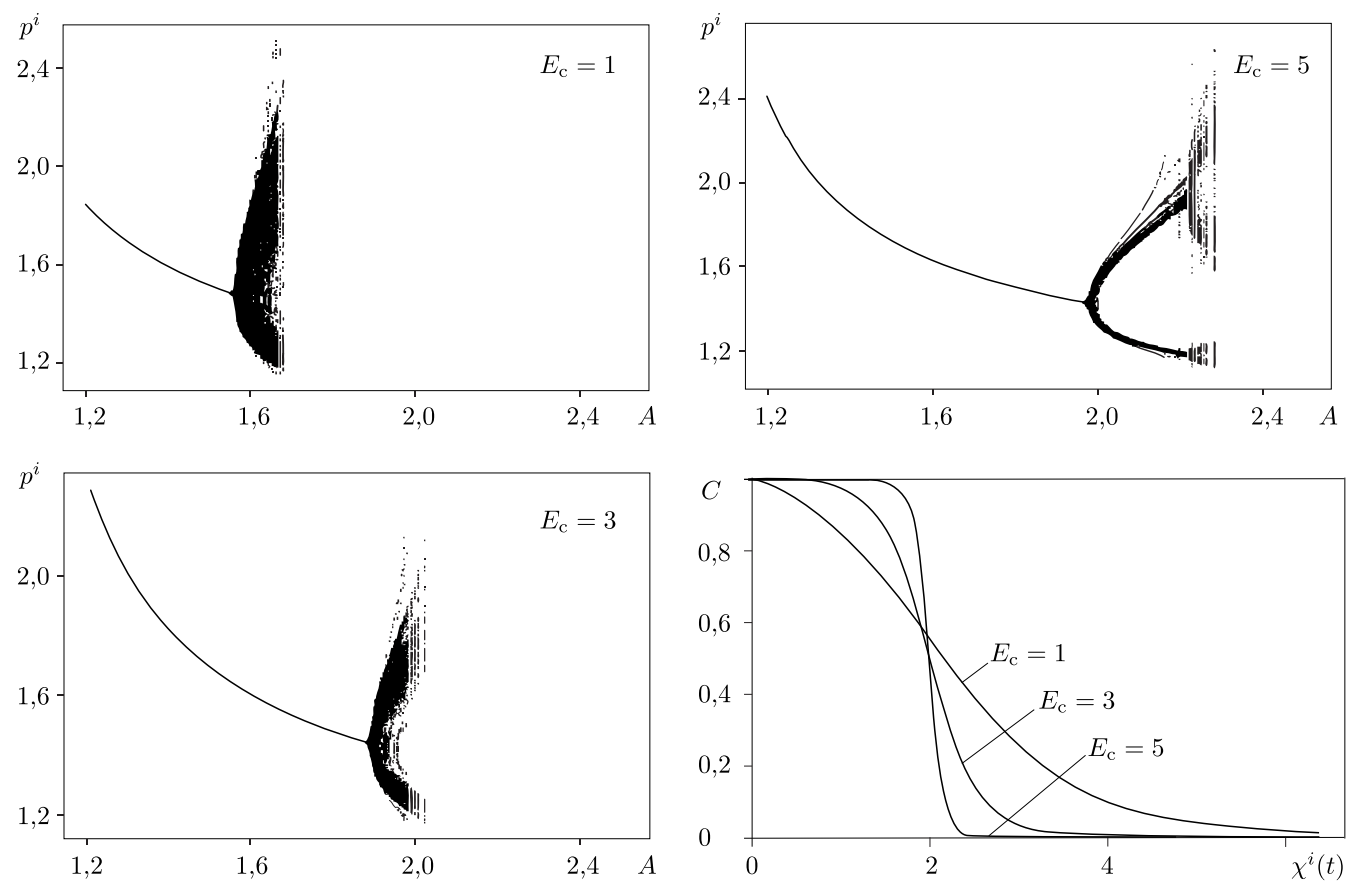

Рис. 2. Сценарии перехода к хаосу системы (6) при малом радиусе корреляции $k=3$
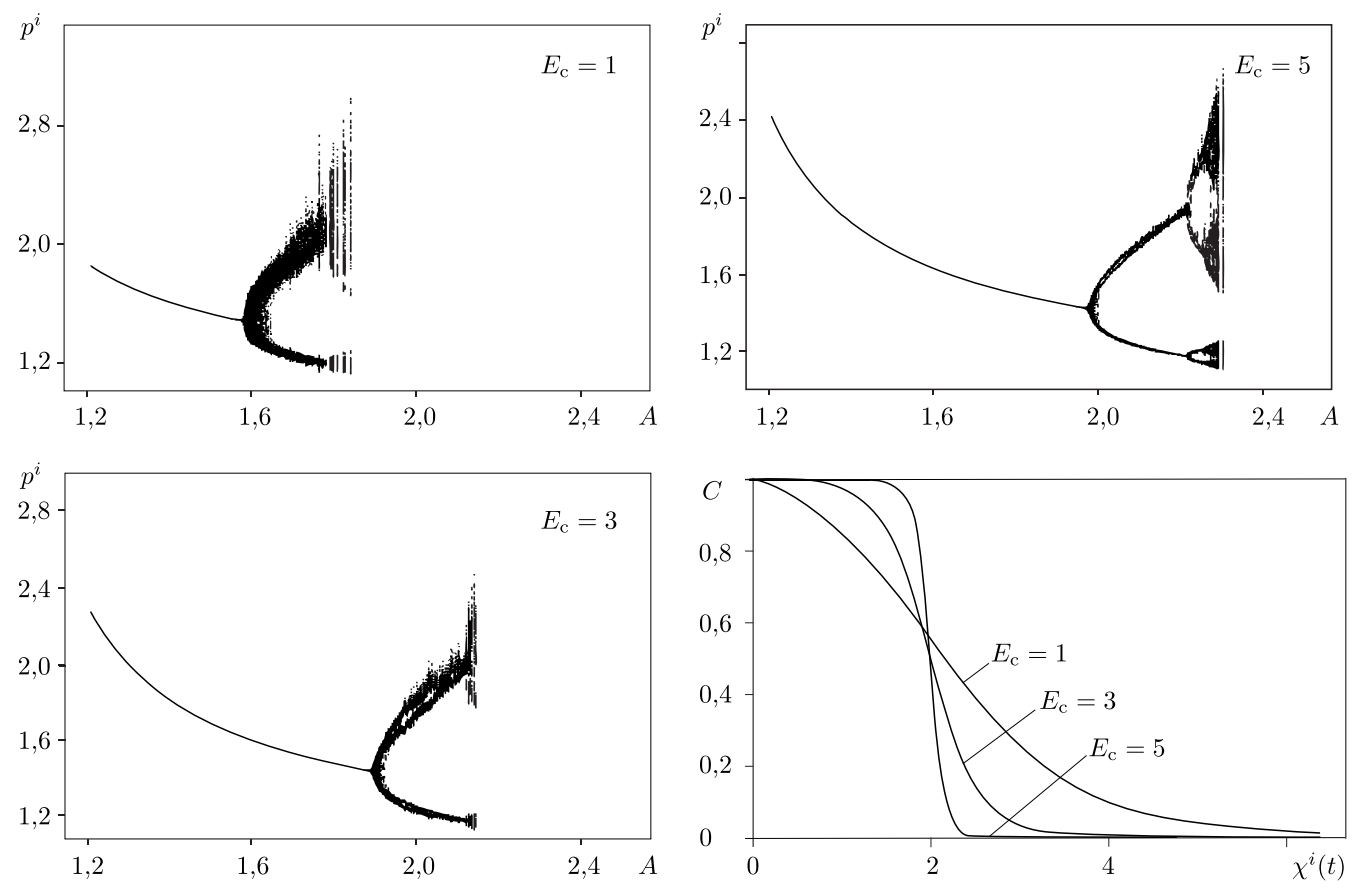

Рис. 3. Сценарии перехода к хаосу системы (6) при большом радиусе корреляции $k=45$ 
Для модели ценообразования (6) проведены численные эксперименты при числе рынков $N=100$ и случайном начальном значении цены, распределённом равномерно на отрезке $[2 ; 3]$. На рис. 2 и 3 показан характер изменения фазопараметрических диаграмм в зависимости от эластичности спроса $E_{\mathrm{c}}$ при фиксированном радиусе информированности $k=3$ и $k=45$ (т.е. при доле доступной информации, равной $7 \%$ и $90 \%$ ) соответственно. Как видно из рисунков, фазопараметрические диаграммы отличаются как сценарием перехода системы к хаосу, так и положением точки бифуркации, в которой система теряет состояние устойчивого равновесия.

При больших $k$ и $E_{\text {с }}$ система переходит к хаосу через бесконечный каскад бифуркаций удвоения периода, и в системе отсутствует зависимость цены от

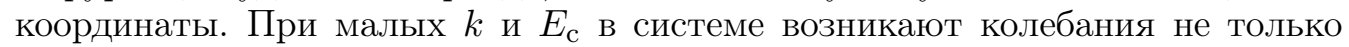
во времени, но также и по координате (номеру рынка). Ясно, что для такой системы приближение одномерной модели уже неприменимо. Качественные изменения поведения системы наблюдаются в следующих интервалах параметров: $1 \leqslant E_{\mathrm{c}} \leqslant 12$ и $1 \leqslant k \leqslant 45$. Численное исследование распределений спроса и цены по номеру рынка позволило выделить область параметров, в которой отсутствует разница между ценами на данных рынках, и показало, что можно пользоваться классической системой уравнений. В рассматриваемой прямоугольной области ей примерно соответствует $k \geqslant 20+32\left(E_{\mathrm{c}}-1\right)^{-1}$.

3. Заключение. В работе предложена новая многокомпонентная модель для исследования устойчивости рыночных механизмов в условиях ограниченного доступа к информации. Неполная информированность агентов учитывается за счёт введения радиуса корреляции между отдельными рынками $k$, а степень влияния данной информации корректируется эластичностью спроса $E_{\mathrm{c}}$. Показано, что при больших значениях $k$ и $E_{\mathrm{c}}$ отсутствует зависимость цен от пространственной координаты и система переходит к хаосу через бесконечный каскад бифуркаций удвоения периода. Такое поведение может быть описано классическим одномерным уравнением. При уменьшении данных параметров в системе возникает пространственная неоднородность, а именно образуются нерегулярные стоячие волны, что требует многокомпонентного описания. Кроме того, при этом качественно меняется сценарий перехода системы к хаосу и положение точки бифуркации. Для рассматриваемого конкретного случая была найдена область значений констант $k$ и $E_{\mathrm{c}}$, в которой система колебательно теряет устойчивость. С экономической точки зрения такое поведение системы означает, что при данном значении эластичности спроса существует критическое значение объема доступной информации, меньше которого любые прогнозы на основе одномерных моделей рыночного равновесия становятся неэффективными.

\section{БИБЛИОГРАФИЧЕСКИЙ СПИСОК}

1. Баутин Н.Н., Леонтович Е. А. Методы и приёмы качественного исследования динамических систем на плоскости. М.: Наука, 1990. 488 с. [Bautin N. N., Leontovich E. A. Methods and rules for the qualitative study of dynamical systems on the plane. Nauka: Moscow, 1990. 488 pp.]

2. Гальперин В. М., Игнатъев С. М., Моргунов В. И. Микроэкономика: В 2-х томах. Т. 1 / ред. В. М. Гальперин. СПб.: Экономич. шк., 2002. 349 с. [Halperin V. M., Ignatiev S. M., Morgunov V.I. Microeconomics. In 2 vols. Vol. $1 /$ ed. V. M. Halperin. St. Petersburg: Ékonomich. shk., 2002. 349 pp.] 
3. Поддубный B. В. Оптимальная стабилизация рынка, описываемого модифицированной динамической моделью Вальраса-Маршалла в пространстве переменных «предложение - цена - спрос»// Вестн. Томск. гос. ун-та, 2004. № 284. C. 80-89. [Poddubny V. V. Optimal stabilization of the market described by the modified Walras-Marshall dynamical model in the space of variables "suggestion - price - demand" // Vestn. Tomsk. Gos. Un-ta, 2004. no. 284. Pp. 80-89].

4. Шананин A. А. Об устойчивости рыночных механизмов // Матем. моделирование, 1991. T. 3, no. 2. Pp. 42-62. [Shananin A. A. On the stability of market mechanisms // Matem. Mod., 1991. Vol. 3, no. 2. Pp. 42-62].

Поступила в редакцию 07/III/2012; в окончательном варианте - 07/VI/2012.

\section{MSC: 91B55; 34C23}

\section{BIFURCATIONS IN A MULTICOMPONENT WALRAS-TYPE SYSTEM UNDER LACK OF INFORMATION}

\section{A. S. Pivovarova}

S. P. Korolyov Samara State Aerospace University

(National Research University),

34, Moskovskoe sh., Samara, 443086, Russia.

E-mail: a-pivovarova@mail.ru

We consider a Walras-type equilibrium model with a supply function specified by A. A. Shananin. In order to investigate a stability of market equilibrium under lack of information we develop a distributed model where price becomes discrete space variable. A bifurcation analysis allows us to examine asymptotic behavior of solutions to the model. We show that in a certain range of parameter values the system becomes spatially unstable and exhibits a qualitatively different bifurcation type.

Key words: Walras equilibrium model, distributed dynamical system, bifurcation analysis, stability of asymptotic solutions, stability of market equilibrium.

Original article submitted $07 / \mathrm{III} / 2012$; revision submitted $07 / \mathrm{VI} / 2012$.

Anna S. Pivovarova, Postgraduate Student, Dept. of Physics. 